\title{
Biphasic Anaphylaxis: A Review of the Incidence, Characteristics and Predictors
}

\author{
Anne K. Ellis* \\ Division of Allergy \& Immunology, Department of Medicine, Queen's University, Kingston, ON, Canada
}

\begin{abstract}
While it has been recognized for over a quarter century that anaphylactic reactions have the potential to follow a biphasic course, reports on the incidence of biphasic anaphylaxis are conflicting, and the search for reliable predictive factors of such responses has been challenging. Further adding to the complexity of this clinical entity are the widely variable durations of the asymptomatic window, and the similarly variable reports on second phase severity. This review aims to provide the health care professional with a better understanding of the true incidence, nature, and risk factors for this type of reactivity by consolidating and summarizing the available literature on the topic of biphasic anaphylaxis. As our body of evidence builds, patterns are emerging to suggest that those patients with an initial presentation requiring more than one dose of epinephrine, those who have life-threatening initial presenting features, and those who otherwise take longer to stabilize, are in this higher risk group, and would be more likely to benefit from prolonged in hospital observation. Conversely, patients who respond rapidly to the immediate administration of epinephrine may be at lower risk, but this finding requires confirmation by others. Further prospective evaluations of biphasic anaphylaxis will greatly aid our understanding of this condition.
\end{abstract}

Keywords: Anaphylaxis, biphasic, late phase response, predictors, allergy.

\section{INTRODUCTION}

Anaphylaxis is a serious allergic reaction that is rapid in onset and potentially fatal [1]. It results from immunologically induced mast cell and/or basophil mediator release after exposure to specific antigen in previously sensitized persons [2]. A recent epidemiological review indicated that anaphylaxis occurs in $1.2 \%$ to $15 \%$ of the US population, and that an estimated 1500 deaths a year may be attributed to anaphylaxis [3].

Variants of the usual monophasic anaphylaxis syndrome include late onset anaphylaxis, biphasic anaphylaxis, and protracted anaphylaxis [4]. While biphasic reactions are a recognized complication, many updated reviews on the topic of anaphylaxis fail to mention the potential for a second phase of reactivity [5-7], or significantly downplay the possibility [8]. Yet as time has progressed, this entity is increasingly being described and acknowledged in the medical literature [9], and additionally is being described as a complication that occurs more frequently than has been traditionally recognized [10,11].

This review will provide an overview and update on the latest research findings in the literature on the entity of biphasic anaphylaxis, with particular emphasize on its clinical characteristics, the incidence rate, and risk factors or predictors of these types of reactions. Articles were identified for inclusion in this review via a MEDLINE search of the literature for studies published between January 1970 and

*Address correspondence to this author at the Division of Allergy \& Immunology, Doran 1, Kingston General Hospital, 76 Stuart Street, Kingston, ON K7L 2V7, Canada; Tel: 613.548.2336; Fax: 613.546.3079; E-mail: ellisa@queensu.ca
December 2009 on biphasic response using the keywords anaphylaxis, biphasic, biphasic anaphylaxis, and late phase reaction. Review articles identified also underwent a reference search for other publications of relevance. Studies published in abstract form only were not included as per the restrictions of this journal.

\section{EPIDEMIOLOGY AND INCIDENCE OF BIPHASIC ANAPHYLAXIS}

The first case reports of biphasic anaphylactic episodes were reported by Popa and Lerner [12]. They described three individuals who, after successful treatment and resolution of symptoms, experienced a second phase of anaphylactic reactivity that onset after an asymptomatic window of 3 to 4 hours.

Since this case series was published, several retrospective series were reported suggesting that the incidence rate of these biphasic reactions were generally low (Table 1). Douglas et al. performed a retrospective analysis of the records from their outpatient service of patients receiving immunotherapy in addition to a chart review of emergency department (ED) and hospital admissions for anaphylaxis [13]. They described an overall incidence rate for these reactions of $5.8 \%$. Subsequent retrospective analyses continued to report relatively low incidence rates, such as $2 \%$ (from Cianferoni et al.'s review of 113 inpatients [14]), 3\% (from Brady et al.'s ED review [15]), and 6\% (from Lee and Greenes pediatric inpatient series [16]). The most recently published retrospective analyses further suggests relatively low incidence rates of $5.3 \%$ and $11 \%$ for biphasic responses $[17,18]$.

The only retrospective series with an appreciably higher reported incidence was from Brazil and MacNamara's re- 
view of 34 patients admitted for observation after an anaphylactic reaction that "required treatment with adrenaline" [19]. They observed in this cohort of potentially more severe anaphylactic episodes an incidence rate of $18 \%$ for biphasic responses.

However, when one looks at the prospective analysis that have been completed - i.e. when subjects with anaphylaxis are followed carefully forward in time after their first wave of reactivity, higher incidence rates are reported. The first such analyses came from Stark and Sullivan in 1986, who documented a $20 \%$ overall rate of biphasic responses in a cohort of 25 patients identified prospectively from ED visits and hospitalizations [4]. The next such similarly designed study was not published until 20 years later by Ellis and Day, whose prospective series of all anaphylactic responses occurring in a single tertiary care centre in Canada documented an incidence rate of $19.4 \%$ in all-comers with anaphylaxis [20]. This study included both anaphylactic events treated in the ED and discharged home as well as those admitted to hospital and those inpatients suffering an in-hospital anaphylactic reaction due to a medication, etc. The most recent prospective evaluation of biphasic reactions was in the targeted population of immunotherapy-induced anaphylaxis. Scranton et $\mathrm{al}$. prospectively enrolled all subjects experiencing a systemic reaction after immunotherapy injections requiring treatment with epinephrine, and contacted all subjects 24 hours after discharge from clinic to survey their recurrent/ongoing symptomatology, if any [21]. Twenty-three percent $(23 \%)$ of subjects who experienced systemic reactivity in the clinic after an immunotherapy injection had recurrent symptoms, but not all of these symptoms would normally have been classified as an anaphylactic reaction, per se (e.g. generalized itching, malaise). Conversely, both the Stark and Sullivan [4] and the Ellis and Day [20] studies required a minimum of 2 organ systems to be involved in order to be classified as a 'biphasic anaphylactic response'.

Regardless, the true incidence rate for biphasic reactions likely lies between 10 to $20 \%$, depending on how one defines a biphasic response. What may be more important to the practicing clinician, however, is to have a better understanding of which of these biphasic reactions are likely to be life-threatening, and leads to the next part of this review.

\section{CLINICAL CHARACTERISTICS OF BIPHASIC ANAPHYLACTIC REACTIONS}

In reviewing all of the published series of biphasic anaphylactic reactions, it is clear that the severity of biphasic reactions is variable. While the majority of second phase reactions are equivalent to, or milder than, the initial phase of reactivity, a significant minority still presents with lifethreatening features and/or requires more aggressive therapy than the original reaction. In 1992, Sampson et al. reported of a case series of fatal and near fatal food-induced anaphylaxis, of which all three of the biphasic reactions proved to be fatal [22].

Of relevance to determining the optimal post-anaphylaxis observation period [11] is the duration of the asymptomatic window in biphasic anaphylactic reactions. Based on Stark and Sullivan's, and Popa and Lerner's original reports (1$8 \mathrm{hrs}$ and $3-4 \mathrm{hrs}$, respectively) [4,12], the most common rec- ommendation in treatment guidelines is to observe patients for between four to six hours prior to discharge from the ED $[1,23,24]$. However, many cases have now been described where the onset of biphasic reactivity was considerably beyond 4 to 6 hrs, sometimes exceptionally so. In Brazil and MacNamara's series [19], all but one of the biphasic reactions occurred later than this window (range of 9h-29.5h), and the mean time to onset of the second phase in Ellis and Day's report was $10 \mathrm{~h}$ overall, with $>40 \%$ of subjects having an asymptomatic window of more than 10h [20]; the longest interval was 78 hours. Importantly, 13 of the 19 patients who experienced biphasic reactions (68\%) developed recurrent symptoms after they had been discharged from the ED. The study by Brady et al. included patients who experienced recurrences as late as 26 and 40 hours after discharge from the ED [15]. Many other isolated case reports emphasize this potential for very late onset biphasic reactivity [25-27]. Other ranges and mean/median times to onset are summarized in Table 1.

Further convoluting the available research in this field is the variability in how anaphylaxis and also biphasic anaphylaxis were defined in the various studies. To enhance clarity for the reader, the definitions used by the authors in each of the various studies has been included in Table 1. Because of these differences in inclusion criteria, definitions, and overall study design, one truly cannot 'average' all of these reports together, as they must be weighed and judged by their own merits individually. Table $\mathbf{2}$ attempts to 'homogenize' those reports whereby second phase reactivity met a minimum diagnostic inclusion of involving 2 or more organ systems in order to have been defined as a biphasic anaphylactic response.

The findings from these studies speak to the inherent variability of these responses, and have led some authors to suggest extending post-anaphylactic observation times to 24 hours [19], or as a minimum, to guarantee immediate access to self-injectable epinephrine and emergency medical services for the ensuing 48-72 hours post-discharge from the ED [2].

\section{PREDICTORS OF BIPHASIC REACTIVITY}

The ability to accurately predict the likelihood of a biphasic reaction based upon features attendant with the initial presentation would be an ideal advancement in this field, and would further help to resolve the aforementioned issue of post-treatment observation. Selection of those only at highest risk for recurrence to undergo extended observation would maximize the utility of hospital admission. Unfortunately, no single distinguishing clinical feature has yet to be identified as a universal predictor of biphasic reactivity. With increasing data accumulating via the available case series, however, patterns are emerging that may prove helpful in risk assessment for this entity.

Management strategies and initial severity of reaction seem to be linked importantly to the occurrence of biphasic responses. While not all reports have been able to link severity of the initial presentation directly to an increased risk for biphasic reactivity, several authors have now reported that patients requiring more than one dose of epinephrine and/or higher doses of epinephrine to control symptoms $[18,19,21]$ 
Table 1. Summary of Biphasic Reaction Reports

\begin{tabular}{|c|c|c|c|c|c|c|c|c|}
\hline \multirow[t]{2}{*}{ Authors, Year } & \multirow[t]{2}{*}{ Study Design } & \multirow{2}{*}{$\begin{array}{l}\text { No of Subjects } \\
\text { (Biphasic; } \\
\text { Total) }\end{array}$} & \multirow{2}{*}{$\begin{array}{l}\text { Biphasic } \\
\text { Definition }\end{array}$} & \multirow{2}{*}{$\begin{array}{c}\text { Biphasic } \\
\text { Incidence } \\
\text { Rate }\end{array}$} & \multicolumn{3}{|c|}{ Time to Onset 2nd Phase(hrs): } & \multirow[t]{2}{*}{ Ref. } \\
\hline & & & & & Range & Mean & Median & \\
\hline Popa \& Lerner, 1984 & Case Series & $3 ; 3$ & A & N/A & 3 to 4 & 3.5 & 3.5 & [12] \\
\hline $\begin{array}{c}\text { Stark \& Sullivan, } \\
1986\end{array}$ & Prospective study & $5 ; 25$ & A & $20.0 \%$ & 1 to 8 & CND & $\mathrm{CND}$ & [4] \\
\hline \multirow{3}{*}{ Douglas et al., 1994} & Retrospective chart review & $6 ; 103$ & $\mathrm{C}$ & $5.8 \%$ & & & & [13] \\
\hline & IT Clinic & $2 ; 44$ & $\mathrm{C}$ & $5.0 \%$ & $\begin{array}{l}\text { not de- } \\
\text { scribed }\end{array}$ & $\mathrm{CND}$ & $\mathrm{CND}$ & \\
\hline & ED/Hospitalizations & $4 ; 59$ & $\mathrm{C}$ & $7.0 \%$ & 1 to $72 *$ & 30.3 & 24.0 & \\
\hline Brady et al., 1997 & Retrospective chart review & $2 ; 67$ & $\mathrm{C}$ & $3.0 \%$ & 26 to $40 * *$ & 33.0 & 33.0 & [15] \\
\hline Brady et al., 1997 & Case Report (Single) & $1 ; 1$ & A & N/A & $\sim 1$ & & & [27] \\
\hline $\begin{array}{l}\text { Lee and Greenes, } \\
2000\end{array}$ & Retrospective chart review & $6 ; 108$ & $\mathrm{C}$ & $6.0 \%$ & 1.3 to 28.4 & 10.1 & 7.0 & [16] \\
\hline $\begin{array}{l}\text { Cianferroni et al., } \\
2001\end{array}$ & Retrospective chart review & $2 ; 113$ & CND & $2.0 \%$ & $\begin{array}{l}\text { not de- } \\
\text { scribed }\end{array}$ & CND & CND & [14] \\
\hline Smit et al., 2005 & Retrospective chart review & $15 ; 282$ & CND & $5.3 \%$ & 1.2 to 22.5 & 7.6 & CND & [17] \\
\hline Ellis and Day, 2007 & Prospective study & $19 ; 103$ & $\mathrm{~B}$ & $19.4 \%$ & 1.5 to 38 & 10.1 & 8.5 & [20] \\
\hline Mehr et al., 2009 & Retrospective chart review & $12 ; 109$ & $\mathrm{C}$ & $11.0 \%$ & 1.2 to 20.5 & 9.5 & 8.8 & [18] \\
\hline Scranton et al., 2009 & $\begin{array}{c}\text { Prospective study, IT } \\
\text { clinic }\end{array}$ & $14 ; 60$ & $\mathrm{C}$ & $23.0 \%$ & 2 to 24 & 7.2 & 5.0 & [21] \\
\hline \multicolumn{9}{|c|}{ Abbreviations: $\mathrm{CND}=$ Could not determine; $\mathrm{ED}=$ Emergency Department; IT = Immunotherapy; O/P = out-patient; N/A = Not applicable } \\
\hline \multicolumn{9}{|c|}{ A= Required Hypotension, Laryngeal obstruction, Bronchial Obstruction, and/ or Respiratory Arrest to be present in 2nd phase to be included } \\
\hline
\end{tabular}

have an increased risk of a biphasic anaphylactic response. That many authors have described the majority of biphasic responses to be clinically similar to the initial presentation $[16,19,20]$, is in itself, an indication that a more severe initial reaction would be a risk factor for a biphasic response to itself be severe, were it to occur.

The other emerging theme is that suboptimal management of anaphylaxis may predispose to biphasic responses. Lee and Greenes noted a significant delay in administration of epinephrine amongst those who suffered a biphasic reaction compared to those who did not (190 versus 48 minutes) [16]. In the Ellis and Day series, time to resolution of symptoms after administration of treatment was significantly longer amongst those who developed biphasic reactivity compared to the uniphasic responders (133 min vs. $102 \mathrm{~min}$ ), which may have reflected the other trend they noted towards lower doses of epinephrine and corticosteroids being used in the biphasic group for management of the initial reaction [20]. Whether or not this was a reflection of the underlying severity of the reaction vs. inadequacy of treatment could not be reliably determined based on the sample size, a problem inherent with any study of this nature given the challenge of vigorously studying so many cases of anaphylaxis prospectively. Conversely, Ellis and Day also noted that those pa- tients whose anaphylactic symptoms were completely resolved in under 30 minutes (all of whom received parenteral epinephrine, be it subcutaneous or intramuscular) were universally protected from having a biphasic response [20]. Taken together, these findings suggest that immediate, appropriate administration of epinephrine could help prevent biphasic anaphylaxis, or at the least, risk stratify the need for prolonged observation (i.e. if immediate delivery of $0.3 \mathrm{mg}$ IM epinephrine did not lead to symptom resolution in under half an hour, they were not in the 'protected group', but also, they were more likely to require a second dose, and thus have another identified risk factor for biphasic responses).

A delayed onset to the development of symptoms after the initial exposure to the antigen [4,17], and oral ingestion of antigen $[4,13]$ have been noted as potential predisposing factors in two reports thus far, however, many studies that specifically looked for these risk factors were unable to confirm the same [15,16,18-20]. Scranton et al. reported biphasic reactions after immunotherapy-induced anaphylaxis [21] further confirming that the route of antigen delivery need not be oral to experience a biphasic response. Any other potential risk factors/predictors identified throughout the various case series, such as female gender [21], and higher body temperature [17] have only been described in isolated reports 
Table 2. Biphasic Anaphylaxis Reports (Minimum Criteria of 2 or More Organ Systems Involved for Inclusion)

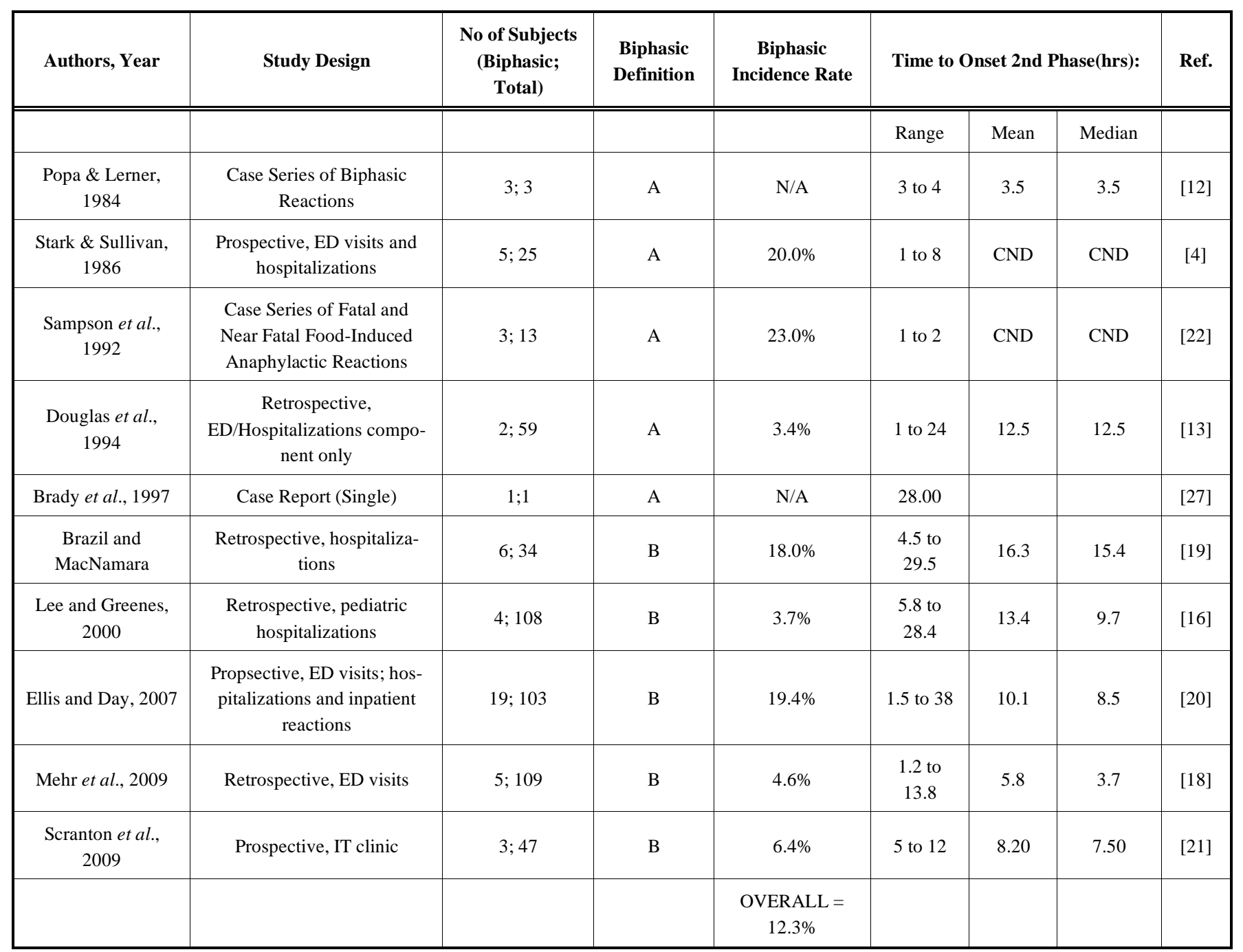

Abbreviations: $\mathrm{CND}=$ Could not determine; $\mathrm{ED}=$ Emergency Department; IT = Immunotherapy; O/P = out-patient; N/A = Not applicable $\mathrm{A}=$ Requires Hypotension, Laryngeal obstruction, Bronchial Obstruction, and/ or Respiratory Arrest to be present in 2nd phase

$\mathrm{B}=$ Requires 2 or more organ systems to be involved

and are unlikely to be as robust as the factors summarized above. Additionally, reports of 'protective' factors have been contradicting, with some studies suggesting that the use of corticosteroids may have been protective against biphasic reactivity $[13,20]$ and others not $[4,15-19]$. Only on isolated occasions has the absence of respiratory symptoms [17] or hypotension [13] been noted to be potentially protective.

\section{CONCLUSIONS}

Biphasic anaphylaxis is a well-recognized variant presentation of the anaphylaxis syndrome. The variability of its clinical features, however, particularly with respect to the time to onset of the second phase and the severity of the biphasic reactions, has resulted in a general under-recognition of its potential clinical importance. Taking into consideration all of the reported case series, with appropriate weighting for study design, suggests that the actual incidence of clinically relevant biphasic reactivity lies between 10 and $20 \%$ of all anaphylactic reactions (i.e. involving more than one organ system with potentially life-threatening manifestations). These reactions can occur much later than previously appreciated, including up to and beyond 24 hours of a symptomfree interval.

Of particular interest to health care providers is the identification of those individuals at highest risk from suffering an adverse outcome if discharged inappropriately early from the ED after initial resolution of symptoms. As our body of evidence builds, patterns are emerging to suggest that those patients with an initial presentation requiring more than one dose of epinephrine (i.e. to stabilize initial symptoms), who have life-threatening initial presenting features, and those who otherwise take longer to stabilize (regardless of management options selected), are in this higher risk group, and would be more likely to benefit from prolonged in hospital observation. Conversely, those who respond rapidly to the immediate administration of epinephrine may be at a much lower risk, but this finding requires confirmation by others. Further prospective evaluations of biphasic anaphylaxis will greatly aid our understanding of this condition. 
All patients being discharged from care post-anaphylaxis, regardless of location, should be provided with an epinephrine auto-injector and instructions on its proper use, and physicians should be assured that the patient has ready and prompt access to emergency medical services for return to the ED if necessary.

\section{REFERENCES}

[1] Sampson HA, Munoz-Furlong A, Campbell RL, et al. Second symposium on the definition and management of anaphylaxis: summary report--Second National Institute of Allergy and Infectious Disease/Food Allergy and Anaphylaxis Network symposium. J Allergy Clin Immunol 2006; 117(2): 391-7.

[2] Ellis AK, Day JH. Diagnosis and management of anaphylaxis. CMAJ 2003; 169(4): 307-11.

[3] Neugut AI, Ghatak AT, Miller RL. Anaphylaxis in the United States: an investigation into its epidemiology. Arch Intern Med 2001; 161(1): 15-21.

[4] Stark BJ, Sullivan TJ, Biphasic and protracted anaphylaxis. J Allergy Clin Immunol 1986; 78: 76-83.

[5] Yunginger JW. Anaphylaxis. Ann Allergy 1992; 69(2): 87-96.

[6] Freeman TM. Anaphylaxis: diagnosis and treatment. Prim Care 1998; 25(4): 809-17.

[7] James JM. Anaphylaxis: multiple etiologies-focused therapy. J Ark Med Soc 1996; 93(6): 281-7.

[8] Ewan PW. Anaphylaxis. BMJ 1998; 316(7142): 1442-5.

[9] Lieberman P. Biphasic anaphylactic reactions. Ann Allergy Asthma Immunol 2005; 95(3): 217-26.

[10] Tole JW, Lieberman P. Biphasic anaphylaxis: review of incidence, clinical predictors, and observation recommendations. Immunol Allergy Clin North Am 2007; 27(2): 309-26, viii.

[11] Kemp SF. The post-anaphylaxis dilemma: how long is long enough to observe a patient after resolution of symptoms? Curr Allergy Asthma Rep 2008; 8(1): 45-8.

[12] Popa VT, Lerner SA. Biphasic systemic anaphylactic reaction: three illustrative cases. Ann Allergy 1984; 53(2): 151-5.

[13] Douglas DM, Sukenick E, Andrade WP, Brown JS. Biphasic systemic anaphylaxis: an inpatient and outpatient study. J Allergy Clin Immunol 1994; 93(6): 977-85.
[14] Cianferoni A, Novembre E, Mugnaini L, et al. Clinical features of acute anaphylaxis in patients admitted to a university hospital: an 11-year retrospective review (1985-1996). Ann Allergy Asthma Immunol 2001; 87(1): 27-32.

[15] Brady WJ, Jr., Luber S, Carter CT, Guertler A, Lindbeck G. Multiphasic anaphylaxis: an uncommon event in the emergency department. Acad Emerg Med 1997; 4(3): 193-7.

[16] Lee JM, Greenes DS. Biphasic anaphylactic reactions in pediatrics. Pediatrics 2000; 106(4): 762-6.

[17] Smit DV, Cameron PA, Rainer TH. Anaphylaxis presentations to an emergency department in Hong Kong: incidence and predictors of biphasic reactions. J Emerg Med 2005; 28(4): 381-8.

[18] Mehr S, Liew WK, Tey D, Tang ML. Clinical predictors for biphasic reactions in children presenting with anaphylaxis. Clin Exp Allergy 2009; 39(9): 1390-7.

[19] Brazil E, MacNamara AF. "Not so immediate" hypersensitivity: the danger of biphasic anaphylactic reactions. J Accid Emerg Med 1998; 15: 252-3.

[20] Ellis AK, Day JH. Incidence and characteristics of biphasic anaphylaxis: a prospective evaluation of 103 patients. Ann Allergy Asthma Immunol 2007; 98(1): 64-9.

[21] Scranton SE, Gonzalez EG, Waibel KH. Incidence and characteristics of biphasic reactions after allergen immunotherapy. J Allergy Clin Immunol 2009; 123(2): 493-8.

[22] Sampson HA, Mendelson L, Rosen JP. Fatal and near-fatal anaphylactic reactions to food in children. N Engl J Med 1992; 327: 380-4.

[23] Soar J. Emergency treatment of anaphylaxis in adults: concise guidance. Clin Med 2009; 9(2): 181-5.

[24] Soar J, Deakin CD, Nolan JP, et al. European Resuscitation Council guidelines for resuscitation 2005. Section 7. Cardiac arrest in special circumstances. Resuscitation 2005; 67 (Suppl 1): S135S170.

[25] Brady WJ, Jr., Bright HL. Occurrence of multiphasic anaphylaxis during a transcontinental air flight. Am J Emerg Med 1999; 17(7): 695-6.

[26] Ellis AK, Day JH. Biphasic anaphylaxis with unusually late onset second phase: A case report. Can J Allergy Clin Immunol 1997; 2(3): 106-9.

[27] Brady WJ, Jr., Luber S, Joyce TP. Multiphasic anaphylaxis: report of a case with prehospital and emergency department considerations. J Emerg Med 1997; 15(4): 477-81.

\begin{tabular}{llr}
\hline Received: December 12, 2009 & Revised: January 28, 2010 Accepted: February 02, 2010
\end{tabular}

(C) Anne K. Ellis; Licensee Bentham Open.

This is an open access article licensed under the terms of the Creative Commons Attribution Non-Commercial License (http://creativecommons.org/licenses/by-nc/3.0/) which permits unrestricted, non-commercial use, distribution and reproduction in any medium, provided the work is properly cited. 